\title{
La estructura socio-ocupacional metropolitana brasileña: diversificación y homogeneidad en los ańos 2000
}

\author{
Alexandre-Magno Alves-Diniz. Pontifícia Universidade Católica de Minas Gerais, \\ Belo Horizonte, Brasil. \\ Jupira Gomes de-Mendonça. Universidade Federal de Minas Gerais, Belo Horizon- \\ te, Brasil. \\ Luciana-Teixeira de-Andrade. Pontifícia Universidade Católica de Minas Gerais, \\ Belo Horizonte, Brasil.
}

RESUMEN | Este artículo examina los cambios experimentados por la estructura socioocupacional de las quince principales regiones metropolitanas de Brasil entre 2000 y 2010. Emplea medidas de ubicación y especialización geográfica para identificar patrones de distribución espacial relacionados con ocho grupos sociales jerárquicos. Los resultados muestran una relativa estabilidad en la composición y distribución de las categorías sociolaborales en las quince regiones metropolitanas, con poca especialización regional, a pesar de los cambios sociales y económicos significativos experimentados durante la década de 2000. Los cambios observados, aunque de corto alcance, están asociados con las metrópolis situadas más al norte.

PALABRAS CLAVE | áreas metropolitanas, desigualdades regionales, transformaciones socioterritoriales.

ABSTRACT | This article seeks to understand the changes undergone by the socio-occupational structure of 15 Brazilian metropolitan regions between 2000 and 2010. It employs location and geographic specialization measures to identify spatial distribution patterns related to eight hierarchical social groups. Results show a relative stability in the composition and distribution of the socio-occupational categories across the 15 metropolitan regions, with little regional specialization, despite the significant social and economic changes experienced during the 2000s. Observed changes, although short ranged, are associated with the northernmost metropolises.

KEYWORDs | metropolitan areas, regional inequalities, socio-territorial transformations. 


\section{Introducción}

Una década es un tiempo corto para observar las alteraciones estructurales de una sociedad. No obstante, es un lapso suficiente para la consolidación de tendencias que provienen de procesos anteriores y para la aparición de nuevas tendencias, tal como lo demuestran algunos procesos recientes. El propósito de este texto es entender las transformaciones que ocurrieron en la estructura socio-ocupacional de las principales regiones metropolitanas (RM) brasileńas entre 2000 y 2010, para lo cual nos basamos en algunas constataciones ya consolidadas en la literatura.

Un primer hecho necesario de tener en cuenta es que la globalización viene alterando de forma significativa las funciones desempeñadas por las grandes RM del Brasil, desencadenando dos procesos contradictorios. Por un lado, la creciente integración del país a los mercados internacionales; y, por otro lado, una agudización de la dispersión y concentración de las actividades productivas, según los moldes propuestos por Henderson (1974), Hirschman (1958), Krugman (1991, 1998) y Myrdal (1957). Destaca, además, que Brasil está hoy incorporado a la economía mundial de una manera específica: es, al mismo tiempo, una potencia financiera emergente (Paulani, 2012) y un país exportador de commodities. Por lo menos desde los años ochenta (Cano, 2011), el país ha sufrido un proceso de relativa desindustrialización y desconcentración de sus áreas industriales. Si, en un primer momento, São Paulo concentró una gran parte del parque industrial brasileño, constituyéndose en el líder en este campo, luego se ha observado una relativa descentralización regional de la industria, proceso al que han contribuido las inversiones en infraestructura y las políticas de incentivo federales o estaduales. En ese sentido, la reversión de la concentración de São Paulo fue acompañada por el crecimiento relativo de otras regiones metropolitanas, señal de un importante cambio en el patrón locacional de la industria brasileña. La caída en la producción industrial fue también fuertemente sentida en la región metropolitana de Río de Janeiro, mientras que otras regiones metropolitanas, como Belo Horizonte, Curitiba y Fortaleza, presentaron un significativo crecimiento en la participación productiva brasileña (Diniz \& Campolina, 2007). Al mismo tiempo, se ha asistido a lo largo de los últimos ańos a una expansión en el sector de servicios superiores, mientras que los servicios personales permanecieron relativamente estables (Biderman \& Lopes, 2015). Particularmente importante a la presente discusión son las ideas emanadas de la Nueva Geografía Económica (Krugman, 1998), que confieren al espacio una gran importancia en la determinación del crecimiento de las regiones. De acuerdo con esta perspectiva, la aglomeración de las actividades productivas en determinado lugar se explica por fuerzas que atraen (centrípetas) y que repelen (centrífugas) tales actividades.

Los años dos mil estuvieron marcados por la consolidación de un modelo de desarrollo económico basado en el reforzamiento del mercado interno a través de la expansión del consumo masivo, asociado a tasas bajas de ahorros (Messa, 2012). Contradiciendo la tendencia de las décadas inmediatamente anteriores, de bajo crecimiento económico, con hiperinflación e inestabilidad económica, la economía brasileńa creció el 3,9\% anual entre 2002 y 2011 (Instituto de Pesquisa Econômica 
Aplicada [IPEA], 2017), catalizada por la valorización del precio de las commodities en el mercado internacional y por el superávit primario creciente (Maia, 2013).

No obstante lo anterior, no existe consenso en la literatura sobre la década del dos mil: por un lado, algunos autores afirman que Brasil vivió un periodo de "nuevo desarrollismo" o, por lo menos, una transición que apunta en esta dirección (Bresser-Pereira, 2011), la cual representaría un alejamiento de las medidas neoliberales que predominaron en América Latina en los ańos 1980 y 1990, fomentando mejoras significativas de las condiciones de vida de la población (Boschetti, 2013). Por otra parte, autores como Cano (2011) y Paulani (2012) consideran que, aunque el periodo pos-2003 haya estado marcado por políticas sociales positivas, desde el punto de vista de la política macroeconómica la orientación neoliberal del periodo anterior se habría mantenido.

De cualquier modo, el dinamismo del mercado laboral generó un crecimiento de la población ocupada, un paso adelante en la formalización de las relaciones de trabajo, un aumento de la fuerza salarial y la reducción de las diferencias de ingresos laborales (Neri, Vaz \& Souza, 2013). El crecimiento económico, que incentivó la creación de empleos, y la política de valorización del salario mínimo han aumentado de forma significativa el poder adquisitivo de los trabajadores. Además, se ha observado la creación y ampliación de políticas de redistribución de los ingresos para los segmentos históricamente excluidos de la población (Instituto Brasileiro de Geografia e Estatistica [IBGE], 2013; Ribeiro, 2012). Como corolario, se ha observado una reducción de la desigualdad de renta salarial en la primera década del siglo.

Con todo, análisis recientes, realizados a partir de informaciones de declaraciones del Impuesto a la Renta obtenidas en la Recaudación Federal brasileña, muestran que la desigualdad es más amplia de lo que se esperaba, eso sin tener en cuenta el hecho de que ella permaneció estable entre 2006 y 2012 (Medeiros, Souza \& Castro, 2015a). La renta ha aumentado para todos, pero los ricos consiguieron apropiarse aún más que antes de este crecimiento. Entre los sectores más ricos, el $0,1 \%$ concentra $11 \%$ de la renta total, mientras que el $1 \%$ concentra $25 \%$ y el 5\%, casi el 44\% (Medeiros, Souza \& Castro, 2015b). La investigación de Morgan (2017) sobre el periodo de 2001 a 2015 confirma esa interpretación, indicando una concentración todavía levemente superior. Ambas investigaciones señalan el origen de la desigualdad estructural del país en la altísima concentración de renta en la cumbre de la pirámide y en las resistencias a cambiar esta realidad, con una muy precaria recaudación de renta y de patrimonio de los más ricos. Además, esa desigualdad extrema se agudiza con las desigualdades de género y de raza, para citar solo dos que todavía son muy persistentes, sobre todo las que tienen que ver con el color de la piel (IPEA, 2011).

La discusión sobre la desigualdad está íntimamente vinculada a la estructura social del país. Para algunos, el cambio más significativo ha sido la aparición de una "nueva clase media" (Neri, 2011). Para otros, lo que sucedió fue un acercamiento de las capas más altas de la clase trabajadora a las capas más bajas de la clase media (Scalon \& Salata, 2012), pero sin un cruzamiento real de la frontera de clase, que no se podría realizar solamente a través de la apropiación de renta-argumento de 
la primera proposición-, sino que por medio de otros atributos (Salata \& Scalon, 2015; Souza, 2010).

Desde el punto de vista macroestructural, observamos reordenamientos sectoriales importantes en la composición del producto interno bruto y en la organización del mercado laboral a lo largo de los años 1990 y 2000. La creciente apertura económica en este periodo permitió, por un lado, la modernización de empresas y, por otro lado, la extinción de compañías menos productivas. Frente a esto, el análisis de la contribución sectorial en la producción de riquezas realizado por Pauli, Nakabashi y Sampaio (2012) para el periodo de 1990 a 2008 demuestra una participación cada vez mayor del sector de servicios, en detrimento de la industria de transformación, que pierde su importancia relativa con la modernización del sector. En estas dos décadas, el sector agropecuario presentó una tendencia poco volátil, a pesar del hecho de que la industria de transformación haya mostrado un desempeño bajo en la creación de empleos (Kaupfer, 2017). Este cuadro confirma lo que encontró Azzoni (1997), al estudiar la evolución regional de los sectores económicos a lo largo de 56 años.

Los estudios comparativos que abordan estas cuestiones en la escala metropolitana son escasos, pese al trabajo de Pasternak (2012), punto de inicio esencial. La autora analizó los impactos de la reestructuración productiva en la estructura socioocupacional de diez regiones metropolitanas y de una aglomeración urbana, entre 1991 y 2000. Según ella, a pesar de sus singularidades, las metrópolis analizadas conservan semejanzas en cuanto al perfil de sus estructuras socio-ocupacionales. En todas las metrópolis, la participación de las ocupaciones medias se destaca -es el caso de las ocupaciones manuales urbanas-, mientras que la participación de las categorías superiores y de los agricultores se ha contraído. El análisis evolutivo (1991-2000) revela pérdidas en la proporción de agricultores y trabajadores de la industria, y un incremento de la proporción de mano de obra ocupada en servicios y en el nivel superior. Desde el punto de vista regional, se observa la reducción de la proporción de dirigentes y de agricultores en las metrópolis del sudeste, y un incremento de las categorías superiores en el noreste. Las capas medias han perdido relevancia en el sureste, mientras que, estas mismas, en el noreste, crecieron en Salvador y bajaron, muy sensiblemente, en Natal, Fortaleza y Recife. En cuanto a las principales regiones metropolitanas, se advierte la expansión de las ocupaciones del sector terciario especializado en Sáo Paulo, Río de Janeiro y Belo Horizonte, conjuntamente con las ocupaciones de nivel superior. En los ańos noventa las metrópolis del sur presentaban mayor estabilidad en sus estructuras socio-ocupacionales, con excepción del incremento de las ocupaciones de nivel superior.

Las transformaciones en la estructura económica son elementos cruciales para la comprensión de las variaciones de la demanda de trabajo y de la dinámica de este mercado y, por ende, de la estructura socio-ocupacional, como se objetiva en el trabajo aquí presentado, que se propone avanzar temporalmente en el análisis. ¿Cómo han ocurrido las recientes transformaciones en el contexto de las regiones metropolitanas brasileńas? Las regiones metropolitanas, ¿han aportado respuestas diferentes a la coyuntura presentada anteriormente? ¿Sería posible hablar de una 
reestructuración productiva o de una recomposición de la estructura socio-ocupacional metropolitana en los años 2000?

El estudio de M. Ribeiro (2016) sobre los espacios metropolitanos brasileños, de modo agregado, establece comparaciones entre las estructuras socio-ocupacionales de 2000 y de 2010, con algunos recortes no metropolitanos, y concluye que no hubo grandes transformaciones en la composición social metropolitana en los años dos mil. Una vez más, el cambio más evidente tiene que ver con el aumento de la participación de las ocupaciones de nivel superior, que, pese a ocurrir en la totalidad del país, fue mayor, en términos proporcionales, en las regiones metropolitanas. A su vez, los otros grupos socio-ocupacionales, según Ribeiro (2016), han experimentado transformaciones modestas en la participación de la estructura social a lo largo de la década, ya que "las alteraciones estructurales no ocurren en periodos tan cortos como aquel de apenas una década" (p. 247). Los datos desagregados para cada región metropolitana, como aquí se han presentado, permiten detallar el análisis y determinar variaciones regionales.

Generalmente, el estudio de la evolución de la proporción sectorial de empleos en los ańos 1990 y 2000 en el país indica una expansión del sector de los servicios (de aproximadamente 10\%), acompañada de la caída de la participación del empleo en los sectores agropecuarios y de la industria de transformación (aproximadamente $6 \%$ y $5 \%$, respectivamente). Sin embargo, es importante destacar una reducción de la industria de transformación entre 1990 y 1998, cuando se estabilizó, volviendo a subir ligeramente después de 2003. El análisis de los datos de forma agregada da cuenta del aumento significativo de la productividad en este periodo -principalmente en la industria de transformación- y de la relativa estabilidad sectorial del empleo y de la generación de riquezas en los años dos mil (Pauli, Nakabashi \& Sampaio, 2012).

El primer decenio del siglo muestra alteraciones significativas en el mercado laboral, con especial atención en el crecimiento del número y la calidad de los empleos creados, además de transformaciones en la estructura ocupacional, con la reducción en la agricultura del número de ocupaciones que requieren menos capacitación y el aumento, principalmente, del número de profesionales y trabajadores en el sector administrativo, ocupaciones típicas de la clase media. No obstante, a pesar de una expansión de la participación de profesionales, técnicos y auxiliares administrativos, el rendimiento medio de estas ocupaciones no siguió la variación de la renta de las otras ocupaciones. El hecho es que los aumentos porcentuales del salario ocurrieron sobre la base de la pirámide ocupacional, fuertemente determinados por la política de valorización continua del salario mínimo -la base de las remuneraciones de las ocupaciones menos cualificadas- y por el incremento de la formalización y el excedente menor de mano de obra (Maia, 2013).

Diniz, Mendonça y Andrade (2018) aportan consideraciones complementarias al estudio de Ribeiro (2016), ofreciendo comparaciones preliminares entre la estructura ocupacional de las quince principales regiones metropolitanas brasileñas en 2000 y 2010, a partir de un análisis estadístico.

En este estudio se trabajaron las regiones de Belém, Belo Horizonte, Campinas, Curitiba, Florianópolis, Fortaleza, Goiânia, Manaus, Porto Alegre, Recife, la Región 
Integrada de Desarrollo Económico del Distrito Federal y Entorno (RIDE-DF), Río de Janeiro, Salvador, São Paulo y Vitória. La RIDE-DF contiene municipios de dos estados, Goiás (19) e Minas Gerais (3), además del propio Distrito Federal. Los autores identifican una expansión significativa de la población ocupada en las RM analizadas, cuyo contingente superó los 30 millones de trabajadores en 2010. Con todo, la distribución de la población ocupada entre las categorías socio-ocupacionales analizadas es heterogénea, revelando una estructura social relativamente diversificada, con una presencia significativa de las ocupaciones medias. Otro aspecto que se destaca es el peso del trabajo manual, que representa, en el conjunto estudiado, casi dos tercios de la población ocupada, proporción que ha disminuido entre 2000 y 2010 (de 61,4\% a 59,0\%).

Con la ayuda de análisis estadísticos, Diniz, Mendonça y Andrade (2018) encontraron diferencias significativas para el periodo 2000 y 2010 en el porcentaje medio de las categorías socio-ocupacionales de dirigentes, pequeños empleadores, ocupaciones de nivel superior y ocupaciones del terciario no especializado. La importancia relativa de las ocupaciones de nivel superior aumentó en un 3,85\% en el conjunto metropolitano, mientras que las categorías dirigentes perdieron puntos $(-0,45 \%)$, así como los pequeños empleadores $(-0,74 \%)$ y las ocupaciones del terciario no especializado $(-2,13 \%)$.

En síntesis, con respecto a las transformaciones observadas entre una década y otra (de 1991 a 2000 y de 2000 a 2010), destaca la caída de los agricultores, lo que revela la continuidad de la urbanización de las RM. Aun así, el relativo descenso de las ocupaciones industriales es parte del proceso de reestructuración productiva que viene ocurriendo desde la década de 1980, y que también se observa en las principales ciudades latinoamericanas (Roberts, 2005). El crecimiento del empleo en el sector de los servicios es el reverso de la medalla de la pérdida verificada en la industria; empero, el crecimiento de las ocupaciones de nivel superior es el cambio más significativo, y se intensifica en la década siguiente (2000-2010), representando incluso la ruptura principal de esta década frente a las otras transformaciones, modestas, en la estructura ocupacional del conjunto metropolitano. Se trata de un resultado proveniente de la expansión de los puestos en la enseñanza pública y privada, de la otorgación de becas de estudio, del crecimiento de los ingresos, así como de la desconcentración espacial de las universidades. En este último caso, es el corolario directo de las políticas públicas en curso en las dos últimas décadas. Si la expansión de la enseñanza privada contribuyó algo en este proceso, son las becas del gobierno y el incremento de los ingresos los factores que han permitido que parcelas significativas de la población, antes excluidas, pudiesen alcanzar el nivel educacional superior. La actual crisis de las universidades privadas, resultante de la reducción de las inscripciones, permite ya observar un retroceso en este proceso, pero los resultados permanecerán todavía por un buen tiempo, sobre todo si tenemos en cuenta el significado de la educación de los padres para la educación de los hijos.

El restringido número de estudios que abordan las transformaciones de la estructura social metropolitana en los años 2000 y el carácter incompleto de sus resultados dejan el campo abierto para análisis comparativos capaces de identificar tendencias generales de concentración espacial de categorías socio-ocupacionales, 
reestructuraciones eventuales y/o especializaciones de regiones metropolitanas relacionadas a tipos ocupacionales específicos. Así, el examen de estas estructuras y de su evolución nos da pistas en lo que se refiere al proceso de desarrollo metropolitano y a las distintas etapas en las cuales cada una de sus unidades se encuentra, midiendo la intensidad de las diferencias entre ellas. Este nuevo ejercicio también puede aportar elementos valiosos para la comprensión de la realidad de la desigualdad socioeconómica, y permite al mismo tiempo estimar la capacidad generadora de ingresos y el potencial de consumo de los trabajadores metropolitanos, ya que la estructura ocupacional es una condición fundamental de la definición de las relaciones sociales y de las oportunidades económicas. Por consiguiente, lo que aquí se intenta hacer es avanzar en el análisis comparativo de la estructura socio-ocupacional de las regiones metropolitanas.

El estudio que presentamos quiere ser una contribución al debate sobre los posibles cambios en la estructura social en las metrópolis brasileñas, materializada mediante el análisis empírico de las diferencias entre las principales regiones metropolitanas respecto de la composición de sus estructuras socio-ocupacionales en dos periodos, 2000 y 2010, basándose para ello en la aplicación de un conjunto de mediciones de localización y especialización geográficas.

El texto a continuación se divide en tres secciones. La primera explica los procedimientos metodológicos, la segunda presenta los resultados, mientras que la tercera se focaliza en las consideraciones finales.

\section{Procedimientos metodológicos}

Existen numerosas propuestas metodológicas para el estudio de la estratificación social disponibles en la literatura. No es el propósito de este artículo presentar y discutir estas diferentes propuestas, y en esa línea nos centraremos en aquellas que estén más relacionadas con la adoptada en este artículo. Una estructura influyente, que ha sido aplicada a otros contextos - destacan al respecto los estudios desarrollados por el Observatorio de las Metrópolis en las regiones metropolitanas brasileñas-, es el cuadro de categorías socioprofesionales (CSP) creado por el Instituto Nacional de Estadística y Estudios Económicos de Francia en 1954 y revistas en 1982. La clasificación tiene en cuenta la naturaleza del trabajo de las personas, destacando su condición, autónoma o asalariada; su posición jerárquica; y la naturaleza del empleador, ya sea privado o público. La clasificación reúne 486 profesiones en 31 categorías socioprofesionales, que a su vez se reparten en seis grupos socioocupacionales (Desrosières, 2008), cómo se discutirá adelante.

El estudio sobre la evolución de la composición socio-ocupacional metropolitana brasileña a lo largo de los años 2000, aquí presentado, tiene como base la metodología definida por el Observatorio de la Metrópolis (Ribeiro, 2000). El punto de partida fue la construcción de una jerarquía social sustentada en la categoría trabajo. La comprensión de esta categoría va más allá de la oposición entre capital y trabajo, abordando distintas posiciones sociales relacionadas con el grado de concentración de capital, posiciones de autonomía o de subordinación, de gerencia o de ejecución, etcétera. Es decir, en el interior de la oposición clásica de clases sociales hay otras 
oposiciones relevantes en el posicionamiento del individuo en la estructura social como un todo. En cada una de esas oposiciones hay un reconocimiento social que posiciona al individuo en una jerarquía: los agentes sociales, como dice Bourdieu (1997), están situados en un lugar del espacio social, lugar que se puede caracterizar por su posición relativa en el conjunto y por la distancia que lo separa de los demás.

Desde el punto de vista empírico, se utilizó la variable 'ocupación' disponible en los Censos Demográficos, definida por el IBGE como la función, el cargo, la profesión o el oficio desempeñado por una persona en una actividad económica, refiriéndose siempre al trabajo principal. A partir de los datos de los censos, se construyó un proxy de la estructura social metropolitana, cruzando la variable ocupación con otras variables, tales como la posición en la ocupación, el sector de actividad, el nivel escolar y de ingresos. Así, los individuos ocupados fueron localizados en posiciones ocupacionales de referencia para la división social del trabajo en vigor en la economía metropolitana brasileńa, para luego identificar los agrupamientos que representan posiciones sociales o clases de posiciones sociales que tengan una cierta homogeneidad social, formando grupos distintos y socialmente reconocibles.

Siguiendo esta metodología, definida por el Observatorio de la Metrópolis (Ribeiro, 2000), las ocupaciones fueran agrupadas inicialmente en 25 categorías socio-ocupacionales representativas del espacio social metropolitano. Esa clasificación dio origen a ocho grupos jerárquicos:

- Dirigentes: Grandes Empleadores, Dirigentes del Sector Público, Dirigentes del Sector Privado.

- Ocupaciones de nivel superior: Profesionales Autónomos de Nivel Superior, Profesionales Empleados de Nivel Superior, Profesionales Estatutarios de Nivel Superior, Profesores de Nivel Superior.

- Pequeños empleadores: Pequeños Empleadores.

- Ocupaciones medias: Ocupaciones Artísticas y Similares, Ocupaciones de Oficina, Ocupaciones de Supervisión, Ocupaciones Técnicas, Ocupaciones Medias de la Salud y de la Educación, Ocupaciones de Seguridad Pública, Justicia y Correos.

- Ocupaciones del terciario: Ocupaciones del Comercio, Prestación de Servicios Especializados.

- Ocupaciones de la industria: Ocupaciones de la Industria Moderna, de la Industria Tradicional, de los Servicios Auxiliares y de la Construcción Civil.

- Ocupaciones del terciario no especializado: Ocupaciones Domésticas, Vendedores Ambulantes y Prestadores de Servicio Precarios.

- Ocupaciones en la agricultura: Ocupaciones Manuales en la Agricultura.

Como contribución metodológica, en este texto se ha trabajado con un conjunto de cálculos de localización y de especialización. Además, la importancia relativa de las categorías en la composición socio-ocupacional metropolitana fue investigada sobre la base del universo de las quince principales regiones metropolitanas brasileñas (Figura 1), cuya definición se basó en estudios llevados a cabo por el IBGE 
(2008) y por Ribeiro (2009), que han identificado y diferenciado, dentro del universo de la RM institucionalizadas, aquellas que tienen efectivamente una naturaleza metropolitana.

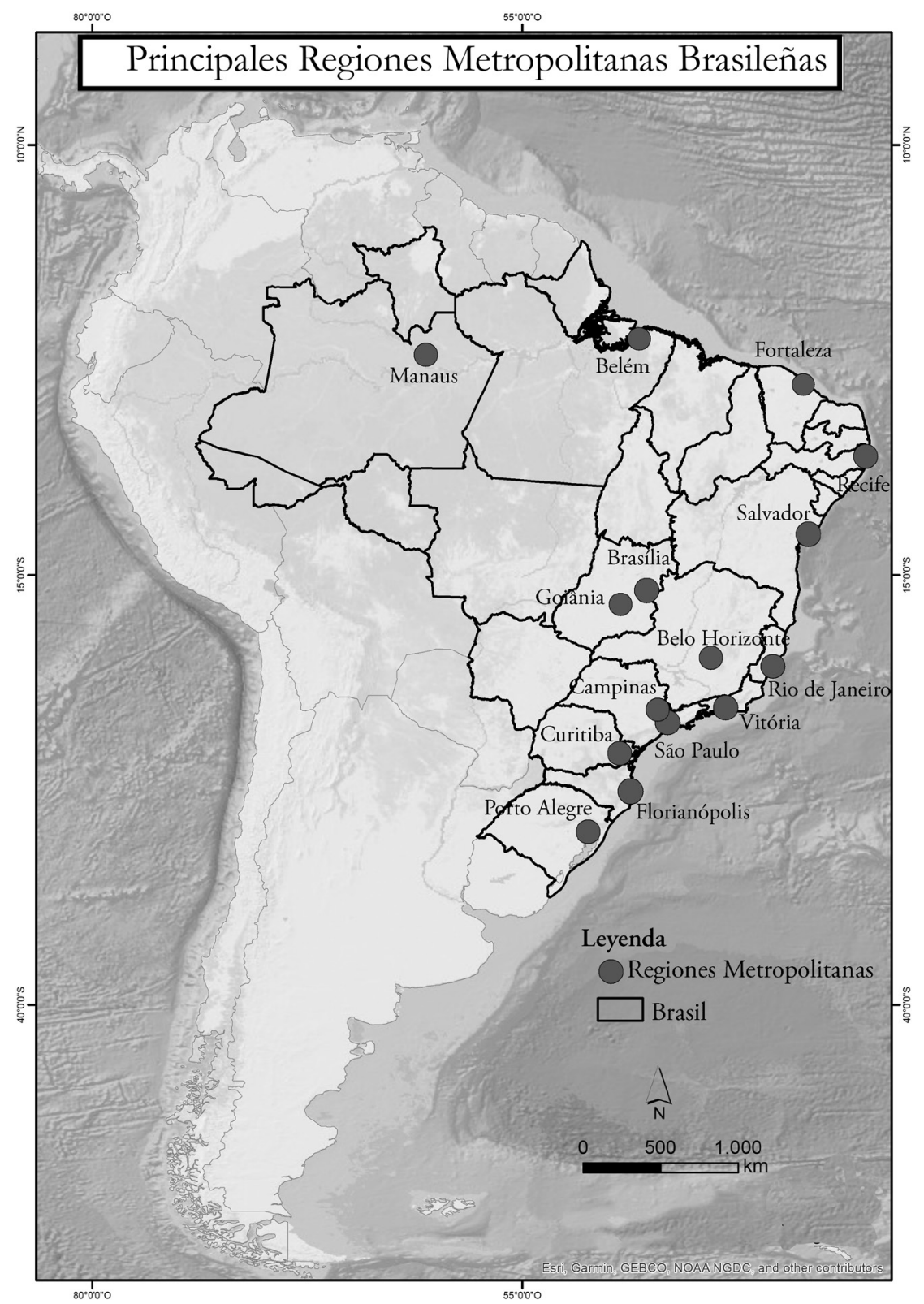

FIGURA I | Principales regiones metropolitanas brasileñas

FUENTE ELABORACIÓN CARTOGRÁfica A. G. LACERDA (20I8) 
El análisis del estándar de distribución sectorial y regional de las categorías socioocupacionales en el universo metropolitano brasileño fue realizado con el incremento de cálculos de localización regional. Muy utilizadas en la Economía Regional, estas mediciones representan un conjunto de técnicas descriptivas y exploratorias normalmente utilizadas en diagnósticos y estudios que objetivan procesos de descentralización productiva, focalizados en la identificación de patrones regionales de distribución espacial de las actividades económicas (Simóes, 2005) -véase Haddad (1989) y Kon (1995) para una discusión detallada de las técnicas-. Cabe señalar que, normalmente, la aplicación de las mediciones de localización es realizada con datos porcentuales de empleo en un determinado sector, como medición de la importancia de aquella actividad o de la dedicación a ella (Paiva, 2006). Sin embargo, en este estudio, el cálculo se basó en informaciones organizadas en una matriz que grafica la distribución de las categorías socio-ocupacionales en las regiones metropolitanas brasileñas. Estos datos ya fueran descritos y analizados por Diniz, Mendonça y Andrade (2018) y, por lo tanto, no serán debatidos en detalle aquí, siendo que el objetivo es avanzar en el análisis del grado de especialización y de concentración del empleo metropolitano entre las principales regiones metropolitanas brasileńas.

\section{La organización socio-ocupacional del espacio metropolitano brasileńo}

En 2000, el conjunto de las quince mayores regiones metropolitanas brasileńas contaba con casi 24 millones de trabajadores ocupados, distribuidos de manera heterogénea entre los grupos socio-ocupacionales. En aquel año, 26,85\% de la fuerza de trabajo se encontraba en ocupaciones medias, seguidas por las ocupaciones del secundario $(22,98 \%)$, del terciario $(19,20 \%)$, del terciario no especializado $(17,55 \%)$, de las ocupaciones de nivel superior $(7,33 \%)$, de dirigentes $(2,27 \%)$, de pequeños empleadores $(2,10 \%)$ y de ocupaciones agrícolas (1,73\%) (IBGE, 2003 y 2011).

A lo largo de los años 2000, periodo marcado por el crecimiento económico y la expansión de las oportunidades laborales, hubo una fuerte expansión de la fuerza de trabajo de las RM estudiadas, de tal modo que, en 2010, el contingente de trabajadores superaba los 30 millones. Mientras que la población brasileña en general creció $12,3 \%$ en el periodo, el aumento de la población ocupada fue de 14,0\%. En las RM analizadas, la población ocupada se incrementó en un $25,7 \%$. Sin embargo, desde el ańo 2000 se perciben algunos cambios en lo que se refiere a la importancia relativa de los grupos ocupacionales. Las ocupaciones medias se mantienen como las de mayor peso, representando $26,62 \%$ de los trabajadores metropolitanos, seguidas de las ocupaciones del secundario $(22,26 \%)$, del terciario $(18,84 \%)$, del terciario no especializado (16,11\%) y del nivel superior (11,44\%) (IBGE, 2003 у 2011).

La división de los trabajadores por ocupaciones muestra una estructura social diversificada, con una presencia significativa de las ocupaciones medias, lo que se contrapone a la imagen de una sociedad dicotómica, dividida entre ricos y pobres. Otro aspecto bastante evidente tiene que ver con el hecho de que la fuerza de trabajo metropolitana está, como lo hemos visto, fuertemente marcada por el 
trabajo manual, confirmando la tendencia señalada por Pasternak (2012) para el periodo 1991 a 2000.

Entre 2000 y 2010, las mayores diferencias en el porcentaje medio de las categorías socio-ocupacionales se observan entre los dirigentes, los pequeños empleadores, las ocupaciones de nivel superior y las ocupaciones del terciario no especializado. La importancia relativa de las ocupaciones de nivel superior aumentó 3,85\% en el conjunto metropolitano, mientras que las categorías de los dirigentes $(-0,45 \%)$, de los pequeńos empleadores $(-0,74 \%)$ y de las ocupaciones del terciario no especializado $(-2,13 \%)$ perdieron importancia. Estos resultados están en sintonía con las alteraciones en la estructura ocupacional metropolitana identificadas por Ribeiro (2016) con base en fuentes de datos y metodología de trabajo diferentes.

\section{Cocientes de localización (CL)}

Una vez identificada la estructura socio-ocupacional del conjunto metropolitano brasileño, se puede realizar el examen de las especificidades regionales. ¿Existen especializaciones productivas inherentes a las quince regiones metropolitanas? Si existen, ¿en qué medida reflejan la estructura socio-ocupacional del conjunto de las regiones metropolitanas? Entre 2000 y 2010, ¿hubo transformaciones de los niveles de especialización regional? ¿Es posible identificar, a la luz de los datos de los años dos mil, alguna alteración en el grado de especialización de las fuerzas productivas en las regiones metropolitanas brasileñas? ¿Cómo desembocan las transformaciones identificadas en la concentración de ocupaciones en el territorio metropolitano?

Una manera de responder a estas indagaciones es a través de los cocientes de localización ( $\mathrm{CL}$ ). Los CL oscilan del cero al infinito, siendo que un $\mathrm{CL}=1$ significa que la región $j$ ya presenta, con respecto a la categoría socio-ocupacional $i$, una proporción equivalente a aquella que se encuentra en el conjunto de las regiones metropolitanas, siendo que no hay sobrerrepresentación o subrepresentación, y refleja la distribución sectorial encontrada en el conjunto regional. Un CL superior a 1 indica una concentración de la actividad socio-ocupacional $i$ en la región metropolitana $j$. Una de las ventajas de esta técnica es que sus resultados permiten un análisis que se focaliza en la concentración de cada grupo socio-ocupacional en cada una de las regiones metropolitanas (Lara, Fiori \& Zanin, 2010).

De este modo, el cociente de localización manifiesta la importancia comparativa de un segmento productivo (categoría socio-ocupacional) para una región metropolitana vis-à-vis el conjunto metropolitano. En otros términos, el CL indica "cuantas veces más" (o menos) una región metropolitana se dedica a una determinada actividad con respecto al conjunto de las regiones metropolitanas que forman la macrorregión de referencia (Paiva, 2006).

El análisis de los CL de las ocupaciones agrícolas indica un desequilibrio mayor dentro de los grupos ocupacionales, siendo que su importancia es expresiva en algunas RM. Como lo hemos visto, las diferencias tienen que ver con la inserción de cada región en la división nacional del trabajo, derivada de la forma en que el país se insertó en la economía mundial, además de aspectos de formación histórica y social locales. 


\begin{tabular}{|c|c|c|c|c|c|c|c|c|c|c|c|c|c|c|c|c|c|}
\hline \multirow{2}{*}{ 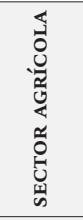 } & 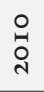 & $\stackrel{\text { సิ }}{\rightarrow}$ & $\begin{array}{l}+1 \\
2 \\
0\end{array}$ & $\stackrel{\infty}{\infty}$ & $\begin{array}{l}\stackrel{*}{n} \\
\hat{i}\end{array}$ & $\stackrel{\infty}{\cong}$ & {$\left[\begin{array}{l}\infty \\
\infty \\
-1\end{array}\right]$} & ले & $\begin{array}{c}R \\
m\end{array}$ & $\begin{array}{l}0 \\
\stackrel{2}{-}\end{array}$ & $\begin{array}{l}\tilde{\sigma} \\
0 \\
-1\end{array}$ & $\begin{array}{c}\tilde{\hat{~}} \\
\mathrm{v}\end{array}$ & $\begin{array}{l}w_{2}^{*} \\
0 \\
0\end{array}$ & $\overrightarrow{\hat{\sigma}^{\prime}}$ & $\begin{array}{l}\text { ?े } \\
\text { o. }\end{array}$ & $\overrightarrow{\hat{\sigma}}$ & \multirow{2}{*}{ 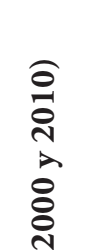 } \\
\hline & \begin{tabular}{l}
$\circ$ \\
$:$ \\
\hdashline
\end{tabular} & $\stackrel{\cong}{=}$ & $\tilde{\sigma}$ & $\underset{\mathbb{Z}}{\stackrel{*}{*}}$ & 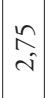 & $\stackrel{\text { ț }}{\rightarrow}$ & $\begin{array}{l}m \\
\hat{i}\end{array}$ & $\stackrel{\nwarrow}{\approx}$ & $\begin{array}{l}\delta \\
\text { f }\end{array}$ & 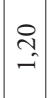 & $\begin{array}{l}\tilde{\gamma} \\
- \\
-1\end{array}$ & $\begin{array}{l}\overrightarrow{\vec{v}} \\
\mathrm{v}\end{array}$ & $\begin{array}{c}2 \\
\tilde{0} \\
0\end{array}$ & $\begin{array}{l}+ \\
\infty \\
0 \\
0\end{array}$ & $\begin{array}{l}\text { ते } \\
\text { ठै }\end{array}$ & 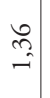 & \\
\hline \multirow{2}{*}{ 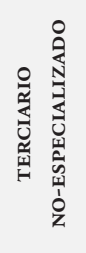 } & $\stackrel{\circ}{\circ}$ & $\exists$ & $\begin{array}{l}2 \\
2 \\
0\end{array}$ & $\tilde{\sigma}_{0}$ & $\begin{array}{l}\stackrel{1}{\infty} \\
0 \\
0\end{array}$ & $\begin{array}{l}n \\
\infty \\
0 \\
0\end{array}$ & $\begin{array}{c}0 \\
- \\
-1\end{array}$ & $\bar{\sigma}$ & $\begin{array}{l}a \\
0 \\
0\end{array}$ & $\mid \begin{array}{l}1+1 \\
0 \\
0\end{array}$ & $\stackrel{\sim}{\approx}$ & $\stackrel{2}{=}$ & $\begin{array}{l}\hat{\theta} \\
-\hat{-}\end{array}$ & $\stackrel{0}{\cong}$ & ळे & $\hat{\sigma}$ & : \\
\hline & ¿ & $\underset{\mathcal{I}}{\approx}$ & 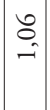 & \&. & $\begin{array}{c}8 \\
0 \\
0\end{array}$ & $\begin{array}{l}\searrow \\
\infty \\
0\end{array}$ & $\stackrel{0}{\cong}$ & $\stackrel{+}{0}$ & $\stackrel{\infty}{-}$ & $\begin{array}{l}\Omega \\
\infty \\
0 \\
0\end{array}$ & $\stackrel{ \pm}{=}$ & $\stackrel{\approx}{\approx}$ & $\begin{array}{c}n \\
\tilde{r} \\
-1\end{array}$ & $\stackrel{2}{=}$ & $\delta$ & $\stackrel{\hat{\sigma}}{-}$ & స్ \\
\hline \multirow{2}{*}{ 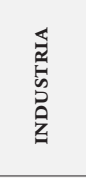 } & $\stackrel{3}{\circ}$ & $\tilde{\sigma}$ & 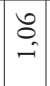 & $\overrightarrow{\mathcal{N}}$ & $\mid \begin{array}{l}\infty \\
0 \\
-1\end{array}$ & $\begin{array}{l}\diamond \\
\infty \\
0\end{array}$ & $\begin{array}{l}\approx \\
\approx\end{array}$ & $\stackrel{ \pm}{=}$ & $\cong$ & $\stackrel{\approx}{\cong}$ & $\begin{array}{l}\hat{\alpha} \\
0 \\
0\end{array}$ & $\begin{array}{l}\infty \\
\vdots \\
0 \\
0\end{array}$ & $\vec{\sigma}$ & $\tilde{\sigma}$ & ळे & $\underset{\sim}{\stackrel{*}{*}}$ & \\
\hline & \begin{tabular}{l}
$\circ$ \\
$:$ \\
\hdashline
\end{tabular} & $\begin{array}{c}\text { के } \\
0 \\
0\end{array}$ & $\begin{array}{l}0 \\
\hat{-} \\
-1\end{array}$ & $\stackrel{\sim}{\approx}$ & $\mid \begin{array}{l}\infty \\
\stackrel{-}{-} \\
-1\end{array}$ & $\begin{array}{l}n \\
\infty \\
0 \\
0\end{array}$ & 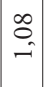 & $\stackrel{\infty}{-\infty}$ & $\hat{\sigma}_{-}^{n}$ & $\overrightarrow{\widehat{N}}$ & $\mid \begin{array}{l}0 \\
\infty \\
0 \\
0\end{array}$ & $\frac{\sqrt{2}}{\hat{0}}$ & $\begin{array}{l}\infty \\
\infty \\
0 \\
0\end{array}$ & $\mid \begin{array}{l}0 \\
\infty \\
0 \\
0\end{array}$ & 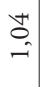 & $\underset{\sigma}{\sigma}$ & \\
\hline \multirow{2}{*}{ 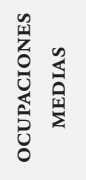 } & $\stackrel{0}{\circ}$ & $\begin{array}{c}\infty \\
\infty \\
0 \\
0\end{array}$ & $\overrightarrow{0}$ & 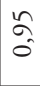 & $\underset{-}{8}$ & $\stackrel{n}{o}$ & {$\left[\begin{array}{c}n \\
0 \\
0 \\
0\end{array}\right]$} & $\tilde{\sigma}$ & $\begin{array}{c}\infty \\
\infty \\
0\end{array}$ & $\mid \begin{array}{l}\infty \\
0 \\
- \\
-1\end{array}$ & $\left(\begin{array}{l}0 \\
0 \\
- \\
-1\end{array}\right.$ & $\mid \begin{array}{l}\infty \\
\hat{\sigma}\end{array}$ & $\stackrel{8}{-}$ & $\begin{array}{l}\tilde{\rho} \\
- \\
-\end{array}$ & $\overbrace{-}$ & & $\stackrel{0}{0}$ \\
\hline & \begin{tabular}{l}
$\circ$ \\
$:$ \\
\hdashline
\end{tabular} & $\begin{array}{l}\infty \\
0 \\
0\end{array}$ & $\begin{array}{l}2 \\
\hat{0}\end{array}$ & $\tilde{\sigma}$ & $\begin{array}{l}\infty \\
\hat{0} \\
0\end{array}$ & $\begin{array}{l}0 \\
= \\
=\end{array}$ & $\left|\begin{array}{l}0 \\
0 \\
0 \\
0\end{array}\right|$ & $\tilde{\sigma}$ & مै & $\begin{array}{l}\infty \\
\tilde{0} \\
0\end{array}$ & $\begin{array}{l}\infty \\
\hat{0} \\
0\end{array}$ & $\begin{array}{c}\tilde{\delta} \\
- \\
-1\end{array}$ & $\begin{array}{c}+1 \\
- \\
-1\end{array}$ & $\begin{array}{c}\infty \\
\tilde{\sigma} \\
-1\end{array}$ & $\begin{array}{l}\mathbb{H}^{\prime} \\
- \\
-\end{array}$ & $\stackrel{\infty}{2}$ & \\
\hline \multirow{2}{*}{ 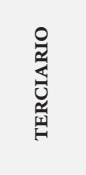 } & 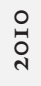 & $\vec{n}$ & $\mid \begin{array}{l}\infty \\
2 \\
0\end{array}$ & $\mid \begin{array}{l}\alpha \\
0 \\
0\end{array}$ & $\mid \begin{array}{c}0 \\
\infty \\
0\end{array}$ & $\begin{array}{l}\tilde{\delta} \\
- \\
-\end{array}$ & $\begin{array}{l}n \\
\hat{n} \\
-1\end{array}$ & $\tilde{\sigma}$ & $\stackrel{\circ}{=}$ & $\mid \begin{array}{l}+1 \\
\infty \\
0\end{array}$ & $\begin{array}{c}\sigma \\
- \\
-\end{array}$ & $\begin{array}{c}+1 \\
0 \\
-1\end{array}$ & $\begin{array}{c}\hat{0} \\
-\end{array}$ & $\mid \begin{array}{l}\infty \\
0 \\
-1\end{array}$ & $\begin{array}{l}2 \\
\vdots \\
0\end{array}$ & $\stackrel{\infty}{\stackrel{\infty}{-}}$ & .0 \\
\hline & : & $\underset{-}{-}$ & $\begin{array}{l}\hat{\alpha} \\
\hat{0}\end{array}$ & $\left|\begin{array}{l}n \\
0 \\
0 \\
0\end{array}\right|$ & $\overrightarrow{\hat{o}}$ & $\hat{\hat{o}}$ & $\begin{array}{l}\hat{\theta} \\
-\end{array}$ & $\hat{\hat{o}}$ & $\hat{\alpha}$ & $\mid \begin{array}{l}\hat{\infty} \\
0 \\
0\end{array}$ & $\begin{array}{l}\exists \\
\approx\end{array}$ & $\hat{\hat{o}}$ & $\stackrel{8}{\circ}$ & $\stackrel{0}{\cong}$ & $\begin{array}{l}\infty \\
0 \\
0\end{array}$ & $\underset{-}{8}$ & \\
\hline \multirow{2}{*}{ 至 } & 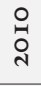 & $\begin{array}{l}\infty \\
\infty \\
0\end{array}$ & $\begin{array}{l}\infty \\
0 \\
0\end{array}$ & $\begin{array}{l}\tilde{\alpha} \\
\hat{0}\end{array}$ & 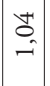 & 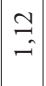 & $\begin{array}{l}\hat{A} \\
\hat{0}\end{array}$ & $\begin{array}{l}\infty \\
\infty \\
0\end{array}$ & $\frac{m}{0}$ & $\begin{array}{l}2 \\
2 \\
0\end{array}$ & $\mid \begin{array}{l}0 \\
0 \\
0 \\
0\end{array}$ & $\hat{\partial}$ & $\begin{array}{l}\infty \\
\stackrel{0}{-}\end{array}$ & {$\left[\begin{array}{c}n \\
\infty \\
0 \\
0\end{array}\right.$} & 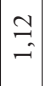 & $\begin{array}{l}\hat{\infty} \\
0 \\
0\end{array}$ & \\
\hline & : & 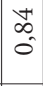 & $\begin{array}{l}\infty \\
0 \\
0\end{array}$ & $\begin{array}{l}n \\
\hat{0}\end{array}$ & 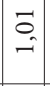 & $\hat{\overbrace{}}$ & $\begin{array}{l}\stackrel{N}{0} \\
\hat{0}\end{array}$ & $\begin{array}{l}\infty \\
\infty \\
0\end{array}$ & $\begin{array}{l}\hat{\tilde{n}} \\
\hat{\sigma}\end{array}$ & $\mid \begin{array}{l}\infty \\
0 \\
0\end{array}$ & $\stackrel{n}{\alpha}$ & $\mid \begin{array}{c}\tilde{O} \\
- \\
-\end{array}$ & $\begin{array}{l}0 \\
= \\
=\end{array}$ & $\mid \begin{array}{l}0 \\
\infty \\
0 \\
0\end{array}$ & $\begin{array}{l}\hat{0} \\
-i\end{array}$ & $\begin{array}{l}n \\
\hat{o}\end{array}$ & \\
\hline \multirow{2}{*}{ 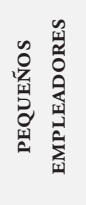 } & $\stackrel{0}{\circ}$ & $\begin{array}{l}8 \\
0 \\
0\end{array}$ & $\mid \begin{array}{l}0 \\
\infty \\
0 \\
0\end{array}$ & $\stackrel{\approx}{\approx}$ & $\stackrel{\hat{\sim}}{=}$ & $\begin{array}{l}\mathfrak{6} \\
- \\
-\end{array}$ & $\begin{array}{l}2 \\
0 \\
0\end{array}$ & $\stackrel{\curvearrowright}{\sim}$ & 定 & 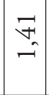 & $\begin{array}{l}\vec{\infty} \\
0\end{array}$ & $\begin{array}{l}\infty \\
\infty \\
0 \\
0\end{array}$ & $\overrightarrow{\hat{\sigma}}$ & $\mid \begin{array}{l}\infty \\
\infty \\
0 \\
0\end{array}$ & $\begin{array}{l}8 \\
-1\end{array}$ & $\begin{array}{l}\triangleq \\
=\end{array}$ & \\
\hline & : & $\begin{array}{l}\text { 苟 } \\
\text { : }\end{array}$ & $\begin{array}{l}\hat{0} \\
\hat{-}\end{array}$ & $\begin{array}{l}0 \\
\approx\end{array}$ & $\begin{array}{l}\tilde{\imath} \\
- \\
-\end{array}$ & $\stackrel{m}{\rightarrow}$ & $\begin{array}{c}0 \\
\hat{0} \\
0\end{array}$ & $\stackrel{\Re}{=}$ & $\begin{array}{l}\tilde{n} \\
\hat{0}\end{array}$ & $\begin{array}{c}\hat{y} \\
=\end{array}$ & $\begin{array}{c}1 \\
0 \\
0 \\
0\end{array}$ & $\tilde{\partial}$ & $\begin{array}{l}+1 \\
0 \\
0\end{array}$ & $\begin{array}{c}\tilde{\sigma} \\
\hat{\sigma}\end{array}$ & $\hat{\sigma}$ & $\overrightarrow{\widetilde{v}}$ & דू \\
\hline \multirow{2}{*}{ 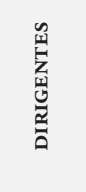 } & 웅 & $\begin{array}{l}\frac{n}{o} \\
0\end{array}$ & \begin{tabular}{l}
\multirow{2}{2}{} \\
0 \\
0
\end{tabular} & $\begin{array}{l}0 \\
0 \\
-\end{array}$ & $\begin{array}{l}0 \\
= \\
=\end{array}$ & $\stackrel{\hat{n}}{=}$ & $\mid \begin{array}{l}* \\
0 \\
0 \\
0\end{array}$ & $\underset{0}{0}$ & S & 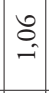 & $\tilde{\sigma}$ & $\stackrel{\infty}{=}$ & 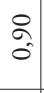 & $\mid \begin{array}{l}0 \\
\infty \\
0 \\
0\end{array}$ & $\exists$ & $\begin{array}{l}\approx \\
\approx\end{array}$ & 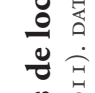 \\
\hline & \begin{tabular}{l}
$\circ$ \\
$:$ \\
\hdashline
\end{tabular} & $\hat{\tilde{\sigma}}$ & $\begin{array}{l}\tilde{\alpha} \\
\hat{\sigma}\end{array}$ & of & $\begin{array}{l}\hat{\theta} \\
- \\
-\end{array}$ & $\overrightarrow{0}$ & {$\left[\begin{array}{l}\infty \\
0 \\
0 \\
0\end{array}\right.$} & $\bar{\sigma}$ & 部 & $\begin{array}{l}\infty \\
0 \\
0\end{array}$ & $\begin{array}{l}\infty \\
\infty \\
0\end{array}$ & $\mid \begin{array}{l}\hat{O} \\
- \\
-\end{array}$ & $\tilde{\sigma}$ & {$\left[\begin{array}{l}0 \\
\infty \\
0\end{array}\right.$} & 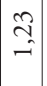 & $\begin{array}{l}2 \\
0 \\
0\end{array}$ & . \\
\hline \multicolumn{2}{|l|}{$\sum_{\approx}^{\Sigma}$} & & 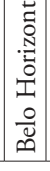 & & 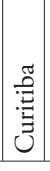 & & 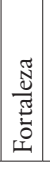 & 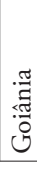 & 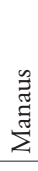 & 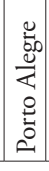 & 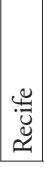 & 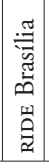 & 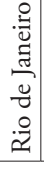 & $\mid \begin{array}{l}\frac{\tilde{n}}{\tilde{n}} \\
\frac{\tilde{s}}{\pi} \\
\tilde{n}\end{array}$ & 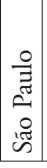 & | & 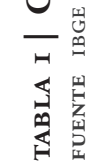 \\
\hline
\end{tabular}


La Tabla 1 permite observar el fuerte agrupamiento relativo de individuos comprometidos en actividades agrícolas en la Región Metropolitana de Manaus, cuya proporción de ocupaciones agrícolas era, en 2000, 4,9 veces mayor que en el conjunto metropolitano estudiado. Este valor permaneció bastante robusto en $2010(3,70)$, indicando la fuerza del sector en la RM de Manaus -donde, a pesar de la concentración industrial y de servicios en la capital, con la Zona Franca de Manaus, la pesca y la agricultura de supervivencia son actividades de relevancia-. Vale señalar que, tanto en 2000 como en 2010, las RM de Curitiba, RIDE-DF, Fortaleza, Goiânia y Florianópolis también presentaron concentraciones de ocupaciones agrícolas superiores a la media metropolitana general. Del otro lado del espectro, tenemos las RM de Río de Janeiro y de São Paulo, metrópolis nacionales por excelencia, que muestran una subrepresentación de las ocupaciones agrícolas en 2000 y 2010, expresión de la esencia urbana, industrial y financiera en el caso de São Paulo, y la importancia de los servicios en el de Río de Janeiro.

En cuanto a la mayor presencia de agricultores, en especial en las RM de Manaus y de Brasilia, cabe resaltar que, en términos absolutos, la participación de esta categoría es muy pequeña. Tal situación aparece confirmada en este artículo al analizarse las ocupaciones en el universo de las quince principales RM, cuya característica más importante es la fuerte presencia del trabajo urbano. Aún se puede destacar algunas desviaciones políticas que han interferido en sus composiciones ocupacionales, como, por ejemplo, las municipalidades pequeñas que fueron incluidas en las regiones metropolitanas por criterios políticos y no por criterios técnicos y en las cuales se concentran las ocupaciones agrícolas) (Tabla 1).

Los dirigentes, a su vez, presentan una importancia regional más equilibrada, en especial en las ciudades de São Paulo, polo nacional y latinoamericano, y de Florianópolis, que despunta como centro de innovación tecnológica con startups y viveros de empresas. En el primer caso, la explicación es histórica, ya que São Paulo siempre se distinguió como una centralidad nacional, pero también latinoamericana (Diniz \& Campolina, 2007; Roberts, 2005). A su vez Florianópolis es un caso reciente, que tiene que ver con la expansión de industrias de tecnologías, con el turismo, así como con la oferta de mano de obra especializada resultante de la fuerte presencia de universidades. Al contrario de São Paulo, la ciudad más industrializada del país y que viene pasando, en las décadas recientes, por un proceso de desconcentración, sin perder su primacía, Florianópolis es un caso que debe ser atribuido a las nuevas tendencias que merecen ser estudiadas (Tabla 1). Por otro lado, tenemos regiones metropolitanas con proporciones de dirigentes por debajo del conjunto metropolitano: las RM de Manaus, Belém y Fortaleza.

Con respecto a los pequeńos empleadores, las regiones metropolitanas del sur de Brasil merecen atención especial, porque presentan concentraciones moderadas de individuos vinculados a ese grupo ocupacional en 2000 y en 2010. Coincidentemente con la subrepresentatividad relativa de la categoría de dirigentes, Fortaleza, Belém y Manaus muestran también una proporción de pequeños empleadores más pequeña que la del conjunto metropolitano (Tabla 1).

El grupo "ocupaciones de nivel superior" está distribuido más uniformemente que los grupos anteriores, destacando Florianópolis en lo que se refiere a esos 
profesionales en 2010. La mayor parte de las RM presentó valores cerca de la norma metropolitana, pero el grupo de las ocupaciones de nivel superior tiene en Fortaleza, Vitória y Manaus una moderada subrepresentatividad (Tabla 1).

Los grupos medios, terciario y terciario no especializado, son los distribuidos de forma más armoniosa en el conjunto metropolitano brasileńo, aspecto confirmado por los CL cerca de 1. La única excepción digna de notarse es la RM de Belém, donde el sector terciario mostró una ligera concentración y donde los profesionales del terciario no especializado ocupaban una posición relevante. Esta región metropolitana viene manteniendo su estructura social "basada en la presencia de actividades terciarias asociadas a la oferta de servicios, cuya mayor parte es vinculada al Gobierno Federal", pero también con una fuerte presencia de establecimientos comerciales de grupos locales, "resquicios del periodo de organización de la economía gomífera del inicio del siglo xx" (Lima, Santos, Sena \& Araújo, 2015, p. 150) (Tabla 1).

Por último, el sector secundario también mostró un alineamiento relativo de las RM con respecto al conjunto metropolitano, salvo Campinas, donde dicho sector estuvo sobrerrepresentado, y la RIDE-DF, donde el sector apareció subrepresentado. Lo que se observa en Campinas es el resultado de un proceso de desconcentración industrial de la Región Metropolitana de São Paulo, que se mantuvo en gran parte dentro del propio Estado. Según C. Diniz (1993), “junto con las deseconomías de aglomeración en el Área Metropolitana de São Paulo, las economías de aglomeración se ampliaron en varias otras ciudades en el Interior mismo de São Paulo y en otros estados. Destácase el eje Belo Horizonte-Porto Alegre, donde está la mayor red urbana del país, la mayor parte de la producción industrial y la mejor infraestructura" (p. 47). De acuerdo con este autor, la Región Metropolitana de Sáo Paulo era responsable, en 1970 , por 44\% de la producción industrial del país, porcentaje que cayó al 26\% en 1990. La participación del Estado en la producción industrial brasileña cayó menos, de 58\% en 1970 al 49\% en 1990, situación que obedeció a la formación de una ciudad-región que reúne cinco regiones metropolitanas (São Paulo, Campinas, Sorocaba, Vale do Paraíba, Litoral Norte y Baixada Santista), dos aglomeraciones urbanas (Jundiaí y Piracicaba) y la microrregión de Bragantina. Este conjunto, juntamente con Río de Janeiro, configura hoy una megarregión que se constituye como el centro del país, en el sentido de "conectar el capital que allí se reproduce con el conjunto de la producción capitalista en la escala global” (Lencioni, 2017) (Tabla 1).

A partir de los CL es posible desarrollar otras conclusiones parciales. En primer lugar, conviene subrayar la existencia de regularidades en el grado de especialización de las fuerzas productivas que constituyen la firma socio-ocupacional de las regiones metropolitanas brasileńas; entre ellas, la presencia equitativa de los grupos ocupacionales medios, secundarios, terciarios y terciarios no especializados.

Otro aspecto discernible a partir de los CL es la conformación de pequeños grupos de regiones metropolitanas con características similares en su constitución socioocupacional. Nos llama la atención el conjunto formado por las RM Belo Horizonte, Salvador, Recife y Vitória, que presentaron CL próximos a la norma general de la RM en todos los grupos ocupacionales; vale decir, son ejemplos emblemáticos de la composición socio-ocupacional básica de la metrópolis brasileña. 
Un segundo grupo estaría formado por las RM de Manaus y Fortaleza, caracterizadas por concentraciones de grupos ocupacionales en actividades agrícolas, situación concomitante con la subrepresentatividad de los grupos dirigentes, de los pequeños empleadores y de las ocupaciones de nivel superior.

Porto Alegre, Curitiba y Florianópolis, las metrópolis del sur, formarían un tercer grupo, en el cual destacan las concentraciones en las actividades agrícolas y de pequeńos empleadores. De hecho, se trata de una región en la cual la fuerte inmigración europea explica la constitución de una tradición agrícola cimentada en la pequeńa propiedad.

Existen algunas regiones metropolitanas que se caracterizan por su excentricidad con respecto al estándar general. Es el caso de Belém, donde los dirigentes y los pequeños empleadores están subrepresentados, pero donde el terciario, como se ha comentado, sobresale. La RIDE-DF, a su vez, exhibe una composición socioocupacional que se caracteriza por la sobrerrepresentación del grupo agrícola y la subrepresentación del secundario. Como capital federal, Brasilia se destaca, desde su inauguración en 1960, como una ciudad con muchos funcionarios públicos y fuerte actividad terciaria, situación que contrasta con la mayoría de los otros municipios de la RIDE. Algunos de estos se constituyen típicamente como ciudades-dormitorio, mientras otros tienen una baja integración con la capital y una fuerte actividad agrícola, en especial la producción de granos para el agronegocio, actividad que empezó a expandirse a partir de los años noventa (Ribeiro, Tenorio \& Holanda, 2015).

Las otras dos metrópolis nacionales, Río de Janeiro y Sáo Paulo, tienen la peculiaridad de poseer una representación del sector agrícola por debajo de aquella del conjunto metropolitano, pese a que São Paulo posee una concentración de dirigentes. Vitória también presentaba una composición desigual en 2000, con una sobrerrepresentatividad del sector agrícola y de los pequeños empleadores y una subrepresentación de las ocupaciones de nivel superior, convergiendo hacia la media nacional de 2010. Finalmente, Campinas fue la única metrópolis con concentración en el grupo industrial.

\section{Coeficientes de Especialización (CE)}

Los CL indicaron cuantas veces mayor o menor es la importancia de cada categoría socio-ocupacional en cada región metropolitana. Pero, si tomamos como referencia la estructura socio-ocupacional de cada RM, en vez de mirar las categorías de forma aislada, y si la comparamos con la estructura socio-ocupacional del conjunto metropolitano, encontramos evidencias con respecto al grado de especialización o de excentricidad de cada región.

El CE ayuda en esta tarea, ya que se trata de una medición regional, que compara la estructura socio-ocupacional de una región metropolitana con la del conjunto metropolitano brasileño. Los procedimientos algebraicos indican que el CE está basado en la suma, en módulos, de las diferencias entre la importancia relativa de la categoría socio-ocupacional $i$ en el empleo total de la región metropolitana $j$ (Sij) y la importancia relativa de la categoría socio-ocupacional $i$ para el conjunto metropolitano $(\mathrm{Si})$. Los valores del CE indican que mientras más cerca de cero sea el 
coeficiente, más parecida será la composición/estructura de la región metropolitana con la del conjunto metropolitano brasileño.

Los valores más cercanos a 1 indican una mayor diferencia con respecto a la estructura socio-ocupacional del conjunto metropolitano, dando cuenta de un grado elevado de especialización productiva (Lara, Fiori \& Zanin, 2010; Lima, Alves, Souza \& Pereira, 2007).

La Figura 2 revela la diversidad de la composición socio-ocupacional de las regiones metropolitanas brasileñas. Mientras que las RM del Sudeste (Belo Horizonte, Vitória y Sáo Paulo) tienen las estructuras ocupacionales que más se acercan al conjunto metropolitano, las metrópolis más septentrionales (Manaus, Belém y Fortaleza) presentan las mayores diferencias. El análisis comparativo de los CE entre 2000 y 2010 nos permitió evaluar el comportamiento del grado de especialización ocupacional de cada región al identificar el mayor acercamiento o distanciamiento de la estructura socio-ocupacional metropolitana global.

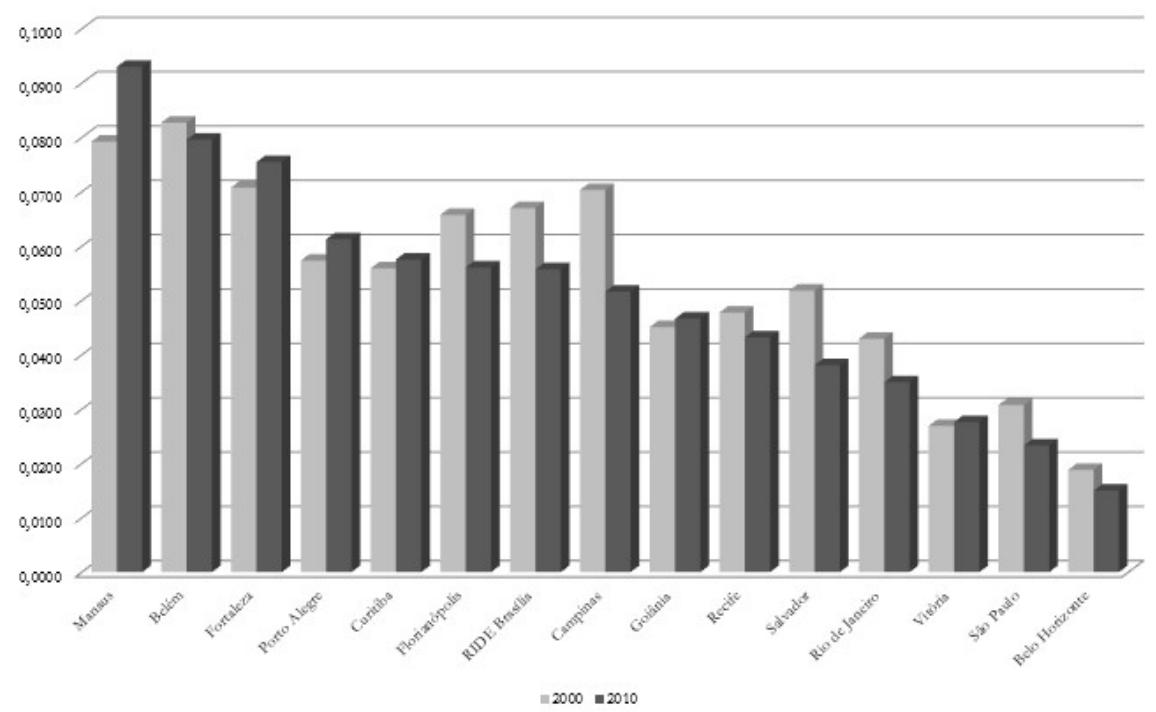

Figura 2 | Coeficiente de Especialización (CE). Categorías Socio-ocupacionales - Regiones Metropolitanas Brasileñas (2000 y 2010)

FUENTE DATOS TRABAJADOS POR LOS AUTORES

Si comparamos los CE de 2000 y de 2010, percibimos una tendencia general de convergencia de las RM brasileñas en dirección a la estructura ocupacional general, lo que se evidencia por la reducción generalizada de los valores de CE en 2010, aspecto que refuerza las tesis de la desconcentración y de la reestructuración productiva en el espacio metropolitano brasileño. Sin embargo, Manaus sobresalta como nota disonante al evolucionar, entre 2000 y 2010, de forma inversa al conjunto metropolitano, como también lo hacen, en cierto modo, Fortaleza y Porto Alegre. 
La estructura socio-ocupacional de Manaus se destaca por la fuerte presencia de profesionales vinculados a los sectores agrícola, secundario y terciario, por encima de la media metropolitana. Fortaleza, a su vez, cuenta con la participación destacada de los profesionales del secundario, del sector agrícola, del terciario y del terciario no especializado. Finalmente, Porto Alegre posee la especificidad de una concentración por sobre la media metropolitana de profesionales en ocupaciones medias y en el sector industrial.

Hasta ese punto, los datos observados muestran que existen diferencias en la estructura productiva de cada metrópolis estudiada, y esto a pesar de la creciente convergencia hacia una estructura más parecida. Como lo hemos analizado, esas diferencias se deben a la formación histórica de cada región metropolitana y a su inserción regional, y su consecuente papel en la estructura urbana brasileńa.

\section{El coeficiente de reestructuración $(\mathbf{C R})$}

El coeficiente de reestructuración (CR) pone en relación la estructura/composición socio-ocupacional de las regiones metropolitanas en dos momentos, en el año base 0 y al año 1 (2000 y 2010), con el objetivo de medir las transformaciones al nivel de sus especializaciones. Los coeficientes iguales a 0 indican la permanencia de las estructuras, mientras que los valores que se acercan a 1 revelan reestructuraciones significativas en la composición socio-ocupacional del periodo en cuestión (Lima et al., 2007).

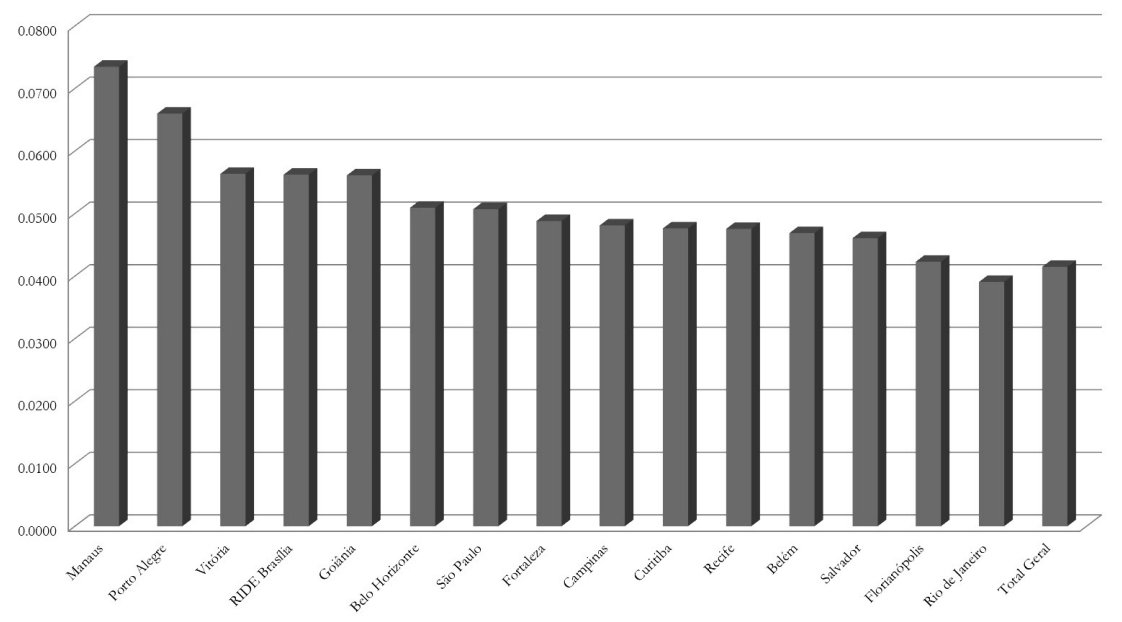

FIgURA 3 | Coeficiente de Reestructuración (CR). Categorías Socioocupacionales - Regiones Metropolitanas Brasileńas (2000 y 2010)

FUENTE DATOS TRABAJADOS POR LOS AUTORES 
Los coeficientes de reestructuración son reveladores de lo poco que se ha alterado la estructura socio-ocupacional metropolitana brasileńa entre 2000 y 2010. Se advierte que el coeficiente para el conjunto metropolitano fue de 0,04 , un valor muy bajo y semejante al de la gran mayoría de las regiones metropolitanas, excepto Manaus y Porto Alegre, las metrópolis que presentaron mayor reestructuración socio-ocupacional en los ańos dos mil, aunque en un grado no demasiado alto (Figura 3). En Manaus se ha observado una expansión del sector superior y del terciario, en detrimento de las ocupaciones vinculadas al terciario no especializado. En el caso de Porto Alegre, también se ha notado una sensible expansión del sector superior, que fue acompañada por la reducción de los sectores terciario y secundario.

\section{Consideraciones finales}

Los datos presentados aquí corroboran una relativa estabilidad en la composición y en la distribución de las categorías socio-ocupacionales entre las quince RM brasileñas en el periodo de 2000 a 2010. Por esta razón, empezamos las conclusiones finales poniendo énfasis en las continuidades, antes de subrayar algunos desvíos identificados en el estándar general, pero afirmando desde ya que la continuidad es la característica más pertinente. Entre ellas (analizadas por el cociente de localización), tenemos una distribución relativamente homogénea entre las RM, de las ocupaciones de nivel superior, de las ocupaciones medias, del sector secundario, del terciario y del terciario no especializado. Además, aunque algunas RM presenten una concentración mayor de algunas categorías socio-ocupacionales, como lo desarrollaremos más adelante, la distribución relativamente homogénea de tantas categorías revela, por un lado, una estructura socio-ocupacional diversificada y, por otro lado, una ausencia de especializaciones regionales fuertes.

La estructura relativamente diversificada y con un contingente significativo de trabajadores en las ocupaciones medias $(26,2 \%)$ pone en tela de juicio ciertos intentos simplificadores del orden socio-ocupacional brasileńo. Asimismo, a pesar de una mayor cualificación de la fuerza de trabajo, proveniente del incremento de los trabajadores con nivel superior entre 2000 y 2010 (de 7,33\% a 11,44\%), esa transformación no cambió la realidad del 59\% de los trabajadores, que se mantuvieron en actividades manuales, signo de la condición y continuidad de un orden socio-ocupacional marcado por esa modalidad de trabajo.

El coeficiente de reestructuración, que pone en relación la composición socioocupacional de las regiones metropolitanas en 2000 y en 2010, confirma el carácter relativamente estable de la estructura socio-ocupacional en esos dos momentos. Los coeficientes indican que el conjunto metropolitano se ha alterado poco en la década, constatación que se extiende también a la gran mayoría de las RM investigadas. Las RM que más han sufrido transformaciones en su estructura fueron Manaus y Porto Alegre, pese al hecho de que los cambios no fueron demasiado significativos.

Así, más allá de la estabilidad estructural identificada en los ańos 2000, el coeficiente de especialización, que compara la estructura socio-ocupacional de una región metropolitana con la del conjunto metropolitano brasileño, indica alguna diversidad entre las regiones metropolitanas, lo que podemos atribuir a una 
formación histórica diferenciada. Así, mientras las RM del sudeste (Belo Horizonte, Vitória, São Paulo y Río de Janeiro) tienen estructuras ocupacionales más próximas al conjunto metropolitano, las metrópolis más septentrionales (Manaus, Belém y Fortaleza) son las que más se alejan de los estándares del conjunto. Por lo tanto, se hace necesario reconocer que, pese a no haberse dado transformaciones estructurales significativas, existen peculiaridades en la composición socio-ocupacional de las regiones metropolitanas estudiadas.

El proceso de desconcentración de la economía industrial y de integración del mercado nacional, particularmente entre los ańos 1970 y 1986, periodo de consolidación de la base industrial del país (Cano, 1997), parece haber producido una convergencia de la estructura socio-ocupacional de las principales regiones metropolitanas brasileńas. La crisis de los años 1980 llevó a un estancamiento del proceso de diversificación industrial. Según Cano (2011), "los segmentos de bienes de producción y de consumo durables se vieron más afectados que los bienes de consumo no durable. Hemos dado un paso atrás en la evolución industrial, en un periodo en el cual el capitalismo mundial aceleraba su reestructuración productiva" (p. 11). Sin embargo, a partir de la década de 1990, los esfuerzos de ampliación de la estructura productiva urbano-industrial en las regiones brasileñas -como lo comprueban, por ejemplo, los incentivos para la instalación de fábricas de vehículos en las regiones Norte, Noreste y Centro-Oeste (Cano, 2012)-, produjeron resultados sólidos desde el punto de vista de la estructura socio-ocupacional.

Es posible observar, entonces, que las diferencias que aparecen en los ańos dos mil, que no son ni tan numerosas ni tan significativas, no logran configurar una especialización perceptible en grupos de regiones o en regiones específicas (salvo, quizá, Florianópolis). Nuestra hipótesis es que las diferencias provienen de factores históricos y regionales y que las similitudes se deben a las políticas estaduales de integración y de descentralización.

De otra parte, por más que São Paulo sobresalga como centro financiero nacional conectado al capital financiero internacional, reuniendo actividades gerenciales, la desconcentración industrial generada por las políticas públicas tuvo el efecto de acercar las metrópolis en estas materias.

Como se destacó al comienzo del texto, si una década es un lapso corto para observar transformaciones en la estructura socio-ocupacional de un país, la tendencia ha sido de una relativa estabilidad, como se infiere del análisis de los datos socio-ocupacionales (1980-1990-2000-2010) realizado periódicamente por el Observatorio de las Metrópolis. ${ }^{1}$ Los logros principales de la década del dos mil están directamente vinculados a los gobiernos de centro-izquierda liderados por el Partido de los Trabajadores (Lula de 2003 a 2011 y Dilma de 2011 a 2016). Se ha hablado mucho de cambios en la esfera social que, de hecho, pudieron ser verificados por medio de indicadores sociales de bienestar y de infraestructura urbana (Andrade, 2016; Arretche, 2015), pero también se habló del nacimiento

1 Véase en Observatório das Metrópoles, Instituto Nacional de Ciência e Tecnologia (https://www. observatoriodasmetropoles.net.br/), la colección "Metrópoles: transformaçôes na ordem urbana" (https://www.observatoriodasmetropoles.net.br/colecao-metropoles-transformacoes-na-ordemurbana/). 
de una nueva clase media y de la reducción de las desigualdades sociales (Neri, 2011), cuestiones que fueron contestadas por otros análisis. Las transformaciones de los indicadores sociales, proceso que ya venía desarrollándose antes del gobierno Lula, siguió, con mejoras significativas en la calidad de vida de los más pobres. El aumento del salario mínimo, la redistribución de la renta por medio de programas como Bolsa Familia, y los esfuerzos en términos de formalización del trabajo, tuvieron un impacto positivo sobre las capas más pauperizadas de la población del país. Sin embargo, como hemos visto, lo que indicaban los estudios anteriores fue que no hubo una reducción de las desigualdades, toda vez que el aumento general de los ingresos del trabajo fue simultáneo a grandes ganancias del estrato que ocupa la cumbre de la pirámide social. Además, el aumento de la renta no se reflejó en la estructura socio-ocupacional, como lo hemos demostrado hasta aquí.

Nuestros resultados con respecto a la estabilidad de la estructura socio-ocupacional van en la misma dirección que los estudios de Scalon y Salata (2012) sobre la estructura de clases. Estos autores disienten de aquella tesis que, basándose solamente en la renta, argumenta que una parcela de la clase trabajadora accedió a la condición de clase media. Según los autores citados -a cuya posición adherimos-, la transformación que se ha observado ha sido mucho más modesta; esto es, se ha dado un pequeño movimiento ascendente, pero en el interior mismo de la clase trabajadora.

Los resultados expuestos confirman la tesis según la cual la estructura social brasileña sigue muy hermética a movimientos mayores que pudiesen ser comprendidos como transformaciones de clases sociales o, como en el caso aquí analizado, de la estructura socio-ocupacional. Sí hay transformaciones, pero todas de corto alcance.

No se ha observado tampoco un alineamiento de las RM brasileńas con un modelo de economía global, como en las ciudades investigadas por Sassen (1991). El terciario especializado y el no especializado, por ejemplo, clasificados como las ocupaciones que más tienen que ver con la economía globalizada, no crecieron en la última década. Al contrario, sufrieron pequeñas caídas. Eso no significa un distanciamiento de la dinámica global, sino más bien una especie de inserción que tiene que ver, entre otros factores, con la realidad de las metrópolis latinoamericanas, muy poco contempladas en los análisis de la globalización (Roberts, 2005).

Los datos analizados nos llevan a proponer la hipótesis, para futuros estudios, de que el proceso de desarrollo brasileńo, después de la crisis económica de los ańos ochenta, no avanzó hacia a una mayor robustez industrial y tecnológica. La opción por las políticas neoliberales a partir de la década de 1990 colocó -paradójicamente- al país en la economía mundial en calidad de exportador de commodities y como un lugar de grandes lucros financieros. En una larga medida, eso explica la estabilidad de la estructura socio-ocupacional de las principales regiones metropolitanas, que regionalmente avanzó en la dirección de la homogeneidad a través de un proceso que parece haberse construido en el periodo de consolidación de la estructura industrial, en especial en los años setenta. 


\section{Agradecimientos}

Los autores agradecen el apoyo de la FAPEMIG (Fundación de Amparo a la Investigación del Estado de Minas Gerais), cnpq (Consejo Nacional de Desarrollo Científico y Tecnológico), Observatorio de las Metrópolis y del investigador Marcelo Ribeiro.

\section{Referencias bibliográficas}

Andrade, L. (2016). Espaço metropolitano no Brasil: nova ordem espacial? Caderno CRH (Online), 29(07), 101-118. http://dx.doi.org/10.1590/S0103-49792016000100007

Arretche, M. (Org.). (2015). Apresentação. En Trajetórias das desigualdades: como o Brasil mudou nos últimos cinquenta anos. São Paulo: Unesp/CEM.

Azzoni, C. (1997). Concentração regional e dispersão das rendas per capita estaduais: análise a partir de séries históricas estaduais de PIB, 1939-1995. Estudos Econômicos, 27(3), 341-393.

Biderman, C. \& Lopes, M. (2015). The geographic dynamics of industry employment in Brazilian metropolitan areas: lessons for São Paulo. Brazilian Journal of Political Economy, 35(3), 492-509. https://doi.org/10.1590/0101-31572015v35n03a07

Boschetti, I. (2013). Políticas de desenvolvimento econômico e implicaçóes para as políticas sociais. SER social, 15(33), 261-384. https://doi.org/10.26512/ser_social. v15i33.13053

Bourdieu, P. (1997). Efeitos de lugar. En P. Bourdieu (Coord.), A miséria do mundo (pp. 159166). Petrópolis: Vozes. [Versión digitalizada en https://bit.ly/3eylm2T].

Bresser-Pereira, L. (2011). O Brasil e o novo desenvolvimentismo. Interesse Nacional (AbrilJunho), 76-85. http://www.bresserpereira.org.br/Papers/2011/10.26a.Brasil_Novodesenvolvimentismo_InteresseNacional.pdf

Cano, W. (1997). Concentração e desconcentração econômica regional no Brasil: 1970/95. Economia e Sociedade, (8), 101-141.

Cano, W. (2011). Novas determinações sobre as questōes regional e urbana após 1980. Texto para Discussão. IE/unicamp, Campinas, 193, 27-53. Estudos Urbanos e Regionais, 13(2), 27-53. https://doi.org/10.22296/2317-1529.2011v13n2p27

Cano, W. (2012). A desindustrialização no Brasil. Economia e Sociedade, 21, n.spe, 831-851. https://doi.org/10.1590/S0104-06182012000400006

Desrosières, A. (2008). Les catégories socioprofessionnelles. Courrier des statistiques, (125), 1315.

Diniz, C. (1993). Desenvolvimento poligonal no Brasil: nem desconcentração nem contínua polarizaçáo. Revista Nova Economia, 3(1), 35-64. https://revistas.face.ufmg.br/index. php/novaeconomia/article/view/2306/1247

Diniz, C. \& Campolina, B. (2007). A região metropolitana de São Paulo: reestruturação, reespacialização e novas funções. EURE, 33(98), 27-43. http://dx.doi.org/10.4067/ S0250-71612007000100002 
Diniz, A., Mendonça, J. \& Andrade, L. (2018). As regiôes metropolitanas brasileiras e sua estrutura social em uma década de mudanças, 2000-2010. Revista de Geografia e Ordenamento do Território, (15), 143-176. https://doi.org 10.17127/got/2018.15.007

Haddad, P. (1989). Medidas de localização e de especialização. En P. Haddad, C. Ferreira, S. Boisier, S. \& T. Andrade (Orgs.), Economia regional: teorias e métodos de análise (pp. 225-247). Fortaleza: BNB-Etene.

Henderson, J. (1974) The sizes and types of cities. American Economic Review, 64(4), 640-656. https://www.brown.edu/Departments/Economics/Faculty/Matthew_Turner/ec2410/ readings/Henderson_AER_1974.pdf

Hirschman, A. (1958). The strategy of economic development. New Haven and London: Yale University Press.

Instituto Brasileiro de Geografia e Estatística (IBGE). (2003). Censo Demográfico 2000, Características gerais da população. Rio de Janeiro, 2003. www.ibge.gov.br/ home/ estatistica/ populacao/cnso2000/default_populacao.shtm

Instituto Brasileiro de Geografia e Estatística (IBGE). (2008). Regióes de influência das cidades. Rio de Janeiro, 2008. https://www.ibge.gov.br/geociencias/cartas-e-mapas/redesgeograficas $/ 15798$-regioes-de-influencia-das-cidades.html? =\&t=o-que-e

Instituto Brasileiro de Geografia e Estatística (IBGE). (2011). Censo Demográfico, 2010, Características da população e dos domicílios: resultados do universo. Rio de Janeiro, 2011. https:// https://censo2010.ibge.gov.br/resultados.html

Instituto Brasileiro de Geografia e Estatística (IBGE). (2013). Sintese dos Indicadores Sociais: Uma análise das condiçôes de vida da população brasileira. http://www. ibge. gov. br/home/ estatistica/populacao/condicaodevida/indicadoresminimos/sinteseindicsociais2013

Instituto de Pesquisa Econômica Aplicada (IPEA). (2011). Faces da desigualdade de gênero e raça no Brasil. Bonetti, A.; Abreu, M. (Org.). Brasília: IPEA. http://www.ipea.gov.br/portal/ images/stories/PDFs/livros/livros/livro_facesdadesigualdade.pdf

Instituto de Pesquisa Econômica Aplicada (IPEA). (2017). IPEADATA. http://www.ipeadata.gov. br.

Kaupfer, D. (2017). Tecnologia e emprego são realmente antagônicos? En J. Sicsú, L. F. de Paula \& R. Michel, Novo-desenvolvimentismo: um projeto nacional de crescimento como equidades social (Cap. 11). Río de Janeiro: Manole. https://www.researchgate.net/ publication/288823354_TECNOLOGIA_E_EMPREGO_SAO_REALMENTE_ ANTAGONICOS.

Kon, A. (1995). Regióes metropolitanas brasileiras: índices de diferenciação. Relatório de Pesquisa No 5. São Paulo: Escola de Administração de Empresas de São Paulo, Fundaçáo Getúlio Vargas. https://bibliotecadigital.fgv.br/dspace/bitstream/handle/10438/3100/ Rel05-95.pdf?sequence= 1 \&isAllowed $=y$

Krugman, P. (1991). Increasing returns and economic geography? Journal of Political Economy, 99(3), 483-499. https://pr.princeton.edu/pictures/g-k/krugman/krugman-increasing_ returns_1991.pdf

Krugman, P. (1998), What's new about new economic geographic. Oxford Review of Economic Policy, 14(2), 7-17. https://doi.org/10.1093/oxrep/14.2.7

Lara, F. Fiori, T. \& Zanin, V. (2010). Notas sobre medidas de concentração e especialização: um exercício preliminar para o emprego no Rio Grande do Sul. Textos para Discussão FEE, 83, 1-24. 
Lencioni, S. (2017). A Megarregião Rio de Janeiro-São Paulo: metropolização do espaço e integraçãoglobal.Boletim Observatóriodas Metrópoles. http://observatoriodasmetropoles. net.br/wp/megarregiao-rio-de-janeiro-sao-paulo-metropolizacao-do-espaco-eintegracao-global/

Lima, J. F., Alves, L. R., Souza, E. C. \& Pereira, S. M. (2007). Alocação espacial da mãode-obra nos estados do Sudeste brasileiro: apontamentos a partir da análise regional. Pesquisa \& Debate, 18, 2(32), 171-195. https://ken.pucsp.br/rpe/article/view/11798

Lima, J., Santos, R., Sena, L. \& Araújo, C. (2015). Estrutura social e organização social da Região Metropolitana de Belém. En A. Cardoso \& J. Lima, J. (Eds.), Belém: transformaçōes na ordem urbana (pp. 145-172). Rio de Janeiro: Letra Capital: Observatório das Metrópoles. https://livroaberto.ufpa.br/jspui/handle/prefix/378

Maia, A. (2013). Estrutura de ocupações e distribuição de rendimentos: uma análise da experiência brasileira nos anos 2000. Revista de Economia Contemporânea, 17(2), 276301. https://doi.org/10.1590/S1415-98482013000200004

Medeiros, M., Souza, P. \& Castro, F. (2015a). A estabilidade da desigualdade de renda no Brasil, 2006 a 2012: estimativa com dados do imposto de renda e pesquisas domiciliares. Ciência e Saúde Coletiva, 4(20), 971-986. https://doi.org/10.1590/141381232015204.00362014

Medeiros, M., Souza, P. \& Castro, F. (2015b). O topo da distribuição de renda no Brasil: primeiras estimativas com dados tributários e comparação com pesquisas domiciliares, 2006-2012. DADOS, Revista de Ciências Sociais, 58(1), 7-36. http://dx.doi. org/10.1590/00115258201537

Messa, A. (2012) Mudanças estruturais na economia brasileira ao longo da década de 2000. Texto para Discussão, ipea, TD 1770. https://www.ipea.gov.br/portal/index. php?option=com_content\&view=article\&id=15556

Morgan, M. (2017). Extreme and persistent inequality: New evidence for Brazil combing national accounts, surveys and fiscal data, 2001-1015. WID Working Paper Series. http:// wid.world/wp-content/uploads/2017/12/113-Morgan-slides.pdf

Myrdal, G. (1957). Economic theory and underdeveloped regions. Londres: Duckworth

Neri, M. (2011). A nova classe média: o lado brilhante da pirâmide. Río de Janeiro: Saraiva.

Neri, M., Vaz, F. \& Souza, P. (2013). Duas décadas de desigualdade e pobreza no Brasil medidas pela Pnadlibge. Comunicados do IPEA, 159. Río de Janeiro, octubre.

Paiva, C. (2006). Desenvolvimento regional, especialização e suas medidas. Indicadores Econômicos FEE, 34(1), 89-102.

Pasternak, S. (2012) Mudanças na estrutura sócio-ocupacional das metrópoles brasileiras, 1991-2000. Cadernos Metrópole, 14(27), 233-278. https://doi.org/10.1590/14789

Paulani, L. (2012). A inserçáo da economia brasileira no cenário mundial: uma reflexão sobre a situação atual à luz da história. IPEA, Boletim de Economia e Politica Internacional, (10), 89-102. http://repositorio.ipea.gov.br/handle/11058/4554

Pauli, R., Nakabashi, L. \& Sampaio, A. (2012). Mudança estrutural e mercado de trabalho no Brasil. Revista de Economia Política, 32(3), 459-478. http://www.scielo.br/pdf/rep/ v32n3/07.pdf

Ribeiro, C. (2012). Quatro décadas de mobilidade social no Brasil. Revista de Ciências Sociais 55(3), 641-679. https://doi.org/10.1590/S0011-52582012000300003 
Ribeiro, L. (2000). Cidade desigual ou cidade partida? Tendências da metrópole do Rio de Janeiro. En L. Ribeiro (Org.), O futuro das metrópoles: desigualdades e governabilidade (pp. 63-98). Rio de Janeiro: Observatório das Metrópoles-IPPUR/UfrJ-FASE; Editora Revan.

Ribeiro, L. (Org.). (2009). Hierarquização e identificação dos espaços urbanos. Rio de Janeiro: Letra Capital/Observatório das Metrópoles.

Ribeiro, M. (2016). Estrutura social e desigualdade de renda: uma comparação entre os municípios metropolitanos e os não metropolitanos do Brasil entre 2000 e 2010. Revista Brasileira de Estudos de População, 33(2), 237-256. https://doi.org/10.20947/ s0102-30982016a0010

Ribeiro, R., Tenorio, G. \& Holanda, F. (Orgs.), (2015). Brasilia Transformaçôes na ordem urbana. Rio de Janeiro: Letra Capital.

Roberts, B. (2005). Globalization and Latin American cities. International Journal of Urban and Regional Research, 29(1), 110-123. https://doi.org/10.1111/j.14682427.2005.00573.x

Salata, A. \& Scalon, C. (2015). Do Meio à Classe Média: como a "nova classe média" e a "classe média tradicional” percebem sua posição social? Revista Ciências Sociais Unisinos, 51(3), 375-386. https://doi.org/10.4013/csu.2015.51.3.14

Sassen, S. (1991). The global city: New York, London, Tokyo. Princeton, NJ: Princeton University Press.

Scalon, M. \& Salata, A. (2012). Uma nova classe média no Brasil da última década? O debate a partir da perspectiva sociológica. Sociedade e Estado, 27(2), 387-407. https://doi. org/10.1590/S0102-69922012000200009

Simóes, R. (2005). Métodos de análise regional e urbana: diagnóstico aplicado ao planejamento. Belo Horizonte: Cedeplar/Ufmg.

Souza, J. (2010). Os batalhadores brasileiros: Nova classe média ou nova classe trabalhadora? Belo Horizonte: UFMG. 